\title{
EL PROFESORADO ANTE EL APRENDIZAJE 2.0: ASPECTOS CRÍTICOS
}

\author{
Fernando Checa García \\ Euro-Mediterranean University Institute \\ Universidad Complutense de Madrid
}

http://dx.doi.org/10.5209/rev_NOMA.2014.v43.n3.49286

\begin{abstract}
Resumen.- La incorporación de la tecnología en el ámbito educativo es un hecho desde hace varias décadas si bien en los últimos años los cambios detectados en el uso de internet por parte de los alumnos han obligado a los docentes a incorporar herramientas colaborativas como apoyo al aprendizaje. Los entornos sociales y tecnológicos obligan a una formación permanente de los profesores y han permitido que la figura del docente recupere su función de facilitador del aprendizaje, más allá de su función como transmisor de contenidos. El aprendizaje colaborativo que permiten las nuevas herramientas y la incorporación de nuevos currículos en todos los ciclos educativos desarrollan e impulsan una nueva forma de responder ante cuestiones como la virtualidad, la socialización y en suma la respuesta ante la tecnologización.
\end{abstract}

Palabras clave.- Tecnología educativa, Educación 2.0, eLearning

Abstract.- The incorporation of technology in education is a reallity for several decades but in recent years the changes detected in the use of internet by students have forced lecturers and teachers to incorporate collaborative tools to support learning. Social and technological environments require ongoing training of teachers and have allowed the figure of the teacher regain his role as facilitator of learning, beyond its role as a transmitter of contents. Collaborative learning that new tools allow, and the incorporation of new curricula at all educational levels, develop and foster a new way to respond to issues such as virtuality, socialization and, in fact, the response technologization

Keywords.- Education Technology, Education 2.0, eLearning

\section{La incorporación de la tecnología a la educación}

Elena Barberà en La educación en la Red (Barbera, 2004) propone once finalidades de la incorporación de la tecnología a la educación que permiten comprender y establecer una hoja de ruta a seguir en la implantación tecnológica. La tecnología en el plano educativo puede observar las siguientes funciones:

1. Socializadora, buscando la inserción del alumno en la sociedad de la información evitando generar cualquier tipo de brecha digital.

2. Responsabilizadora, centrada en los retos y los compromisos del alumno, asumiendo nuevos métodos de enseñanza-aprendizaje.

3. Informativa, priorizando la búsqueda y consulta de fuentes de todo tipo. 
4. Comunicativa, situada en entornos en los que la audiencia no es simulada y generando una comunicación que llega a los destinatarios y recibe respuesta.

5. Formativa y formadora, ayudando al profesor a la incorporación de elementos de feedback e impulsando al estudiante a obtener los conocimientos marcados en los objetivos previos.

6. Motivadora, centrándose en el alumno como motor y centro del aprendizaje.

7. Evaluadora, alentando los procesos que acrediten el conocimiento adquirido.

8. Organizadora, permitiendo la codificación y sistematización del trabajo del profesor así como la coordinación y reglamentación del trabajo de los alumnos.

9. Analítica, ofreciendo un nuevo método de indagación en los procesos educativos.

10. Innovadora, integrando diferentes medios tecnológicos y generando un trabajo de averiguación constante.

11. Investigadora, sujeta a un método constante de contraste y validación.

La facilidad de acceso a las nuevas herramientas de interacción social permite que los individuos puedan acceder y planificar su propio aprendizaje informal. En este aprendizaje informal y en los recursos pertenecientes a la Web 2.0 podemos englobar los vídeos disponibles en Internet, los podcasts, los blogs que ofrecen y permiten crear y publicar información con rapidez y cualquier recurso que el alumno o el profesor pueda considerar importante. Es la persona la que está creando sus propios recursos y la que los almacena para su uso y su fácil y rápido acceso. Los nuevos alumnos están generando conexiones on line similares a sus conexiones mentales y con esos nuevos nodos, cuyo ejemplo más práctico es la popularización y crecimiento de las redes sociales, los alumnos pueden comunicarse y compartir ideas, recursos y experiencias.

Los grupos de alumnos pueden utilizar estas nuevas herramientas para autoorganizarse, colaborar entre sí y crear conjuntamente, así como para trabajar con un objetivo común: el aprendizaje compartido. El alumno, frente a la actitud reactiva, adquiere una actitud proactiva. De una relativa o escasa implicación en el aprendizaje se avanza a un elevado compromiso y de tener como única meta la superación de la materia o asignatura, se crean metas propias. El alumno tradicional reflexionaba poco sobre sus propias actitudes 0 destrezas. Hoy los nuevos estudiantes tienen consciencia sobre las mismas y desean aplicarlas en su aprendizaje. El mismo entorno educativo pasa de una situación basada en la competitividad a entornos colaborativos donde las destrezas enfocadas en la memoria y la repetición son sustituidas por las enfocadas en la comunicación, así como en la búsqueda, selección, producción y difusión del conocimiento (Bautista et Al, 2006). 
Los denominados "Nativos Digitales" (Prensky, 2004) son una generación que ha crecido con la tecnología, capaces de llevar adelante múltiples labores de forma simultánea. Prensky considera que esta generación, que a veces muestran menores habilidades relacionadas con la atención tradicional, pueden concentrarse simultáneamente en varios medios y tienen una gran habilidad para detectar cambios en el entorno. Estos alumnos están accediendo a la universidad con un diferente nivel de conocimientos y habilidades que sus compañeros precedentes. Muy familiarizados con Internet y conectados a través de redes sociales y muy sociables: "Ser amigo de un amigo es aceptable" (Franklin, 2007) prefieren el trabajo en grupo y el aprendizaje experimental. La cuestión final es si estas habilidades y preferencias están siendo afrontadas por los nuevos medios de enseñanza. Podemos plantearnos que el hecho de haber visto incorporada la tecnología desde la infancia no significa obligatoriamente que los estudiantes tengan ya todas las habilidades y actitudes para usar por sí solos las tecnologías colaborativas (Anderson, 2007). En ese sentido los avances en los entornos educativos 2.0 han de contar con una formación que incluya aspectos relacionados con la privacidad, la confianza y la seguridad.

Frente a las tradicionales formas de enseñanza, las nuevas herramientas están siendo utilizadas por los profesores, educadores o formadores para lograr que el aprendizaje formal se convierta en un entorno más participativo, social y colaborativo. La transmisión del conocimiento rompe el tradicional esquema lineal de emisor-receptor para integrarse en un círculo abierto a todo tipo de participantes, no sólo los miembros de la clase y no sólo los contenidos citados por el profesor, sino todo aquello que supone una interacción con el entorno y que puede incorporarse en el proceso. Según Cabero (Cabero et Al, 2000), en el aprendizaje colaborativo lo importante no es sólo la interacción y los intercambios de información entre los participantes, sino la naturaleza y el proceso de la actividad, teniendo presente que el individuo logra alcanzar sus objetivos si el resto de los miembros del grupo también lo hacen. Ningún instrumento en sí mismo se convierte por sí solo en educador o motivador, sino que este carácter le es otorgado cuando la persona hace que se utilice con sentido crítico y por posibilitar los entornos de enseñanza-aprendizaje. Hoy la nueva brecha digital se puede producir entre los usuarios de Internet que aprovechan las nuevas posibilidades, creativas y transformadoras, frente a aquellos con un uso más pasivo. Y esto es aplicable cuando son aplicadas a la docencia colaborativa. El colectivo de usuarios más participativos está mejor preparado para aprovechar las ventajas que la red ofrece. De ahí la necesidad de reforzar la formación en los usos más creativos de la red, en la transformación de las actitudes, especialmente de los docentes que no han crecido con el uso cotidiano de Internet (Duart et Al, 2008). Ante la nueva situación siempre pueden aparecer aquellos favorables al cambio, que vean mayores posibilidades para ayudar a los estudiantes, que intuyan nuevas posibilidades de adquirir competencias docentes diferentes, mejorando su carrera e incluso obteniendo un mayor prestigio. Frente a ellos se sitúa el miedo a perder el control de la enseñanza, el esfuerzo añadido, el miedo a lo desconocido y la certidumbre de enfrentarse a un aumento de trabajo al tener que asumir un rol como profesor mucho más complejo (Bautista et Al, 2006). Ciertamente hay un importante número de profesores que no está interesado en las formas de creación participativa de la web y un exceso enfoque 
"tecnocentrista" puede llevar a errar en el concepto de que, tanto todos los profesores como todos los alumnos, pueden ser fácilmente partícipes de las nuevas metodologías. Incluso los pasos que pueden iniciar algunas universidades en la incorporación de los Entornos Virtuales de Aprendizaje como espacios de desarrollo y trabajo on line de sus alumnos podrían ser poco utilizados prefiriendo los espacios propios (Anderson, 2007).

\section{El aprendizaje en entornos $\mathbf{2 . 0}$}

Actualmente existen plataformas que pueden ayudar a integrar las herramientas sociales y colaborativas y que proveen un espacio seguro para los participantes en la acción educativa. Estas deben proveer a los alumnos y profesores un espacio que permita agregar y almacenar el aprendizaje personal y los recursos asociados al trabajo realizado. Herramientas que ofrezcan que el trabajo se desarrolle de forma segura y aprovechando las sinergias del grupo (Coll, Engel, Bush, 2008). Todas ellas necesitan que los individuos puedan adquirir habilidades para gestionar su propio aprendizaje y que ayuden a los grupos participantes a resolver sus problemas, así como incorporar el concepto de la participación activa, el paradigma de "aprender haciendo, participando y colaborando". En los entornos colaborativos se están conformando nuevas comunidades de aprendizaje siguiendo las teorías avanzadas por Wenger (Wenger, 1999) y las comunidades de prácticas, aunque también puede existir algunas veces cierta sospecha de que no todo el trabajo colaborativo cumpla un aprovechamiento pedagógico (Barbera et $\mathrm{Al}$, 2008). Para lograrlo es necesario que en la incorporación de las tecnologías colaborativas se haga hincapié en los procesos de análisis y proyección, no exclusivamente en la búsqueda, el almacenamiento y la consulta de la información.

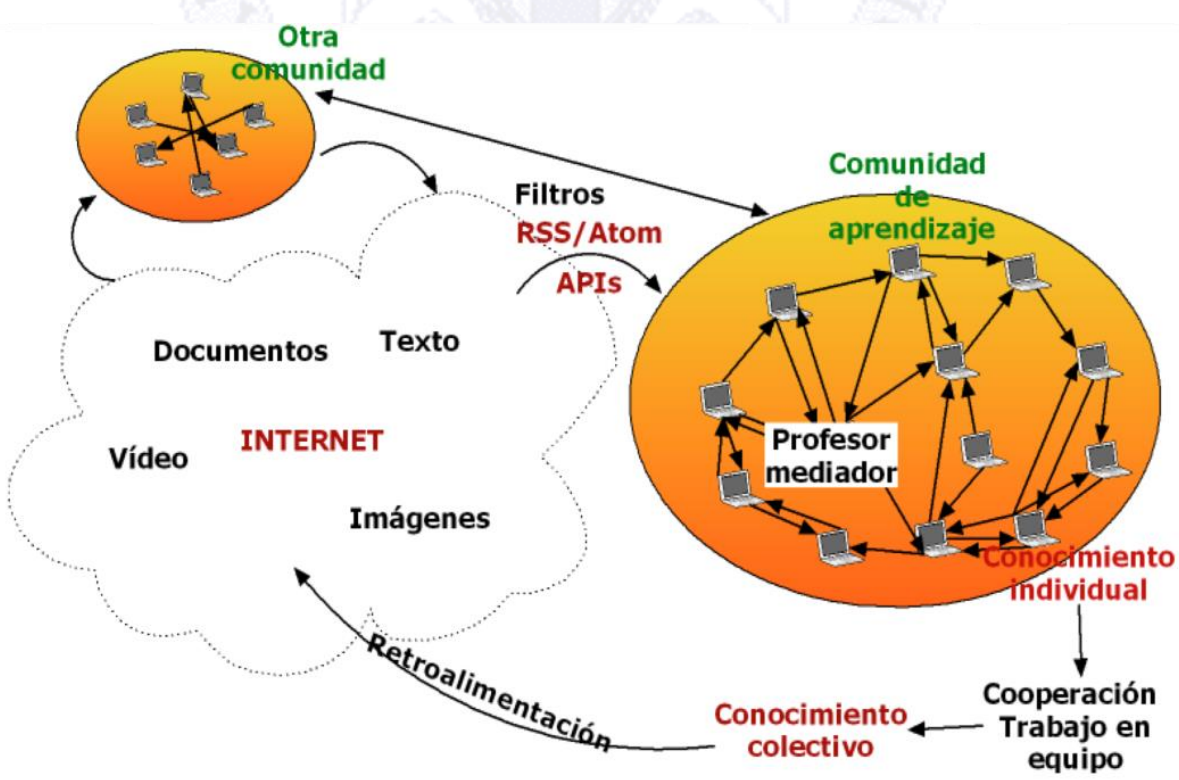

Figura 1. Comunidades de Aprendizaje usando las tecnologías

El término "Aprendizaje 2.0" está íntimamente relacionado con la Web 2.0 y al igual que esta ha cambiado la forma con la que los usuarios se relacionan en 
Internet el "Aprendizaje 2.0" supone una filosofía pedagógica que hace hincapié en entornos de aprendizaje flexibles, prácticos y en muchos casos informales. Los estudiantes adquieren protagonismo al convertirse en creadores de contenido y colaborar con sus compañeros, accediendo a nuevas fuentes de contenido y utilizando las herramientas on line para trabajar conjuntamente y gestionar su propio aprendizaje (Urrutia, 2007), mientras que el profesor ayuda en el aprendizaje proporcionando los apoyos para seguir progresando en todo momento (Coll, Engel, Bush, 2008).

Las comunidades educativas ya se han visto afectadas por la irrupción de la tecnología que ha permitido que las modalidades de formación a distancia se hayan desarrollado especialmente desde el año 2000 haciendo crecer la teleformación o el "e-learning". Este tipo de formación ha ido introduciéndose en los centros educativos ya sea mediante la incorporación de programas de "e-learning" puros o mediante estrategias que lo sumen a la formación tradicional y que apoyen a la "presencialidad", todavía generalizada en los estudios universitarios españoles como indica García Aretio (García Aretio, 2006). Pero el campus físico de las universidades no sólo se está expandiendo hacia la virtualidad sino que Internet y la web colaborativa están generando una mezcla permanente, con escenarios de aprendizaje, físicos o no, y el docente pasa a tener el reto de convertirse en un guía en el acceso a la información, más que en transmisor de la misma (Torre, 2006).

En un entorno de cambio de paradigma formativo un modelo DocenciaAprendizaje 2.0 debe contemplar cuatro principios fundamentales: aprender a aprender, de forma que el alumno sea el que se convierta en el centro de la formación y en protagonista activo del proceso de enseñanza-aprendizaje; el aprendizaje colaborativo, que fomente la participación activa entre alumnos a través de todo un conjunto de distintas actividades, incluidas la creación y participación en comunidades de aprendizaje; en tercer lugar es necesario que los programas formativos sean compatibles y estén conectados con la realidad profesional, de forma que el aprendizaje pueda ser puesto en práctica de forma rápida y tangible. $Y$ de forma transversal, el uso de las Tecnologías de la Información y la Comunicación para mejorar las capacidades de aprendizaje (Castaño Garrido, 2008).

El aprendizaje ha de contar con un desarrollo previo de un plan de formación, así como un diseño completo de los instrumentos y las herramientas que llevarán a su obtención, tanto de los contenidos como al entrenamiento en las competencias profesionales consideradas fundamentales para el mejor desempeño del alumno. En la orientación del nuevo sistema de enseñanzaaprendizaje son también claves las acciones que ofrezcan al alumno la posibilidad de realizar un trabajo independiente y autónomo, que le permita la asunción de responsabilidades y el control de su propio aprendizaje.

El trabajo colaborativo ha de ser el segundo aspecto crítico del modelo, de forma que los alumnos puedan compartir su aprendizaje con el resto de los compañeros del grupo así con el entorno representado por la comunidad de internautas, de forma que la sensación de pertenencia a una comunidad se vea reforzada (Erickson, Kellogg, 2001). 
En tercer lugar es necesario no olvidar el escenario en el que el aprendizaje se produce. En la formación presencial este escenario suele ser el aula, las salas de trabajo, los espacios para las tutorías o los laboratorios. Pero también los entornos de esparcimiento, como las cafeterías de los centros educativos o todos aquellos lugares en los que se produzca interacción entre los alumnos y entre los alumnos y los profesores. Además de los escenarios de "presencialidad" nuestro modelo considera que ha de incorporar las plataformas de teleformación y los Entornos Virtuales de Aprendizaje, uniéndoles espacios de comunicación en entornos abiertos (Esteban, 2003), como blogs, microblogs, wikis y redes sociales, de forma que el alumno pueda conformar su propio espacio personal de aprendizaje o PLE (Personal Learning Environment). Las aplicaciones de la Web 2.0 aplicadas a la docencia permiten la creación y generación de contenidos individuales o colectivos, usar los formatos audiovisuales, incorporar con sencillez material fotográfico y debido a los avances en los desarrollos con AJAX y Flash las respuestas de los interfaces de usuario son similares a las aplicaciones de escritorio. En ellas se pone un esfuerzo especial en la usabilidad y en la simplificación de las interacciones (Ulrich et $\mathrm{Al}, 2008$ ). Los nuevos PLE no son una colección preconstruida de herramientas y contenidos sino un marco que permite al estudiante generar su propio "suite" de aplicaciones y recursos.

En el desarrollo de la planificación de la asignatura es necesario que exista una definición clara de los objetivos y las metas que se quiere alcanzar, una secuencia de los contenidos así como la realización de esquemas conceptuales y de la temporalización de la acción formativa o el curso. De forma paralela es necesario plantear con el tiempo suficiente las actividades que van a realizar los alumnos y realizar una correcta explicación de las mismas, de lo que se pretende lograr con ellas así de las diferentes formas posibles de afrontarlas. En ese sentido también es necesario seleccionar los recursos y las herramientas que van a usarse para la comunicación y la participación, presentar de forma clara por adelantado los criterios que se van a utilizar para llevar a cabo la evaluación. Esta planificación, incorporada desde hace tiempo en la planificación en los cursos de la modalidad de teleformación, se ha demostrado muy efectiva y puede incorporarse al modelo que proponemos (García Aguilera et Al, 2004).

De nuevo siguiendo a Barberà, es necesario establecer un sistema que permita evaluar los aspectos tecnológicos utilizados a modo de checklist con los siguientes aspectos:

1. Concepción, planificación y enfoque global. Creando en esta primera fase los marcos de actuación tecnológicos, pedagógicos y las bases generales de actuación.

2. Finalidad, relacionando los propósitos de uso de los recursos de la red con los objetivos de la institución.

3. Preguntas sobre la evaluación, orientadas a obtener información sobre la implementación del proceso así como la puesta en marcha de las distintas actividades. Será necesario fijarse en los aspectos de mejora, los resultados y la planificación, los procesos de implantación así como los desarrollos profesionales y las destrezas computacionales. 
4. Recogida de información, personal, situacional y documental.

5. Análisis contrastando las informaciones resultantes.

6. Generación de resultados, materializando los diversos productos que puedan haberse obtenido.

7. Comunicación, preparando la publicad interna y externa a la organización.

8. Metaevaluación, generando procesos de control sobre los propios procesos evaluativos.

\section{El profesorado ante el nuevo entorno}

El profesor en el nuevo paradigma ha de incorporar competencias diferentes a su perfil como formador. Estas competencias deben incluir al menos tres bloques:

- Experto. Conocedor de su área de conocimiento, con dominio de su materia y conocimiento del diseño y estructura académica del programa que siguen sus alumnos. Planificador y organizador así como evaluador del desempeño y de los objetivos alcanzados, uniendo a todo el proceso creatividad e innovación.

- Orientador. Guía del proceso de aprendizaje de sus alumnos, sumando estrategias y técnicas de motivación así como actuando como dinamizador y generador de nuevos retos a los alumnos.

- Facilitador tecnológico. Usuario de herramientas que permitan el acceso a la comunicación a través de la red y consejero de sus alumnos de los mejores recursos que incrementen las fuentes de aprendizaje.

Margalef y Alvarez proponen en "La formación del profesorado universitario para la innovación en el marco de la integración del Espacio Europeo de Educación Superior" (Margalef, Álvarez, 2005) que las nuevas tecnologías han de integrarse plenamente en la docencia universitaria, en la mejora de los procesos, en la incorporación de recursos y servicios de la Universidad, así como en los intercambios y la colaboración a distancia. Las nuevas herramientas pueden actuar como facilitadores de la creación de redes de trabajo, comunicación e investigación (Lipponen, 2002).

Hoy no podemos justificar la carencia de conocimiento por una falta de información sino más bien el desconocimiento puede venir por un exceso de la misma. La gran cantidad de información puede llevar a navegar entre ella sin rumbo (Cabero et $\mathrm{Al}, 2000$ ). No tiene sentido competir con la memoria que supone Internet sino que resulta mucho más interesante aliarse con ella. De esta forma, el profesorado ha de ser capaz de enseñar a buscar, a seleccionar y evaluar la información disponible en Internet. No se debe caer en el error de realizar un paralelismo entre información y conocimiento ni pensar estrictamente que por estar expuesto a la información el individuo va a estar necesariamente más informado. Los nuevos problemas de la educación no van 
a ser tanto la localización y búsqueda de información sino más bien la selección, la interpretación y la evaluación (Cabero et Al, 2007). El problema no es tanto el acceso a la información como su búsqueda inteligente, el análisis crítico de la misma y su aplicación para la resolución de problemas. La comunicación verbal presencial solamente permite una comunicación de uno a muchos frente a la utilización de las TIC que también favorecen la puesta en práctica de diferentes estrategias de comunicación. Las tecnologías potencian al mismo tiempo un trabajo individual y cooperativo, favorecen las relaciones interpersonales y se modifican las actitudes hacia los contenidos y las actividades docentes desarrolladas con ellos. Cabero y Prendes consideran que las tecnologías se justifican por su poder para crear entornos diferenciados para el aprendizaje, donde el alumno en interacción con las mismas y en la búsqueda de unos objetivos planteados de antemano construye su propio conocimiento, interactuando con la tecnología, con sus compañeros, con el profesor y con el resto de variables del sistema. El saber flexible y reflexivo, en continua construcción crea, en palabras del profesor Cabero, un "espíritu Wik" centrado en la participación y en el que el saber se muestra como no definitivo.

\section{Conclusiones}

Para evitar que las tecnologías se conviertan en un mero añadido hay que modificar también el rol del estudiante y la concepción misma del aprendizaje. El alumno debe lograr competencias que le capaciten para el análisis y la síntesis, la aplicación de los conocimientos y la resolución de problemas, el trabajo en equipo, el dominio de habilidades interpersonales, la capacidad de planificar y gestionar el tiempo, de adaptarse y de ser creativo e imaginativo.

Por otro lado, la formación del profesorado debe incluir la capacitación en Tecnologías de la Información y la Comunicación en el entorno no sólo instrumental, sino también estético y curricular, pragmático y psicológico, como productor y diseñador, evaluador, crítico, organizativo e investigador. Será la capacidad de absorber las nuevas tecnologías y aplicarlas correctamente en el aula uno de los aspectos clave que durante los próximos años tendrá un recorrido crucial en los procesos educativos. Los nuevos currículos, cada vez más enfocados en la mejora de las competencias, se verán beneficiados con ello y el aprendizaje se situará desde un espacio más profundo que el de la mera transmisión de conocimientos inmutables.

\section{Referencias bibliográficas}

ANDERSON, P, (2007) «What is Web 2.0? Ideas, Technologies and implications for education», JISC Technology and Standards Watch, Bristol, BARBERA, E, (2004) La educación en la Red, Paidós, Madrid - (2008) Cómo valorar la calidad de la enseñanza basada en las TIC: pautas e instrumentos de análisis, Grao, Barcelona

BAUTISTA, G, et Al, (2006) Didáctica universitaria en Entornos Virtuales de Enseñanza, Narcea, Madrid

CABERO, J et Al, (2000) Nuevas tecnologías en la formación flexible y a distancia, Kronos, Sevilla

- (2007) Profesor, ¿estamos en el ciberespacio?, Davinci, La Coruña 
CASTAÑO GARRIDO, C, (2008) Prácticas educativas en entornos Web 2.0, Síntesis, Madrid

COLL, C, BUSTOS, A, ENGEL, A, (2008) «Las comunidades virtuales de aprendizaje», Psicología de la educación virtual. Enseñar y aprender con las tecnologías de la Información y la Comunicación, Morata, Madrid, 299-320 DUART, J, et Al, (2008) La Universidad en la Sociedad Red, Barcelona, Ariel ERICKSON, T, KELLOG, W, (2001) «Knowledge Communities: Online Environments for Supporting Knowledge Management and its Social Context», Beyond knowledge management, Hershey, Idea Group, 209-323

ESTEBAN, M, (2003) «Los entornos de aprendizaje abiertos (EAA) » en Red, Revista de Educación a distancia, Universidad de Murcia, número 7

FRANKLIN, T, (2007) Web 2.0 for Content for Learning and Teaching in Higher Education, Mánchester, JISC, 2007. Disponible en línea en: http://www.jisc.ac.uk/media/documents/programmes/digitalrepositories/web2content-learning-and-teaching.pdf

GARCÍA AGUILERA et AI, (2004) Metodología didáctica aplicada: estrategias de intervención pedagógica, Lavante, Málaga

GARCíA ARETIO, L, (2006) Nuevos ambientes de aprendizaje, Bened, Madrid LIPPONEN, L, (2002) «Exploring foundations for computer-supported collaborative learning", en Computer Support for Collaborative Learning, Proceedings of the Conference on Computer Support for Collaborative Learning: Foundations for a CSCL Community, Boulder, Colorado, 72-81

MARGALEF, L, ÁLVAREZ, J.M, (2005) «La formación del profesorado universitario para la innovación en el marco de la integración en el espacio europeo de enseñanza superior », Revista de Educación, MECD, Volumen 337, 389-402

PRENSKY, M, (2004) The emerging online life of the digital native, Games2Train, Nueva York

TORRE, A, (2006) «Web educativa 2.0» en Edutec. Revista Electrónica de Tecnología Educativa, Número 20, Enero. Disponible en línea en: http://www.uib.es/depart/gte/gte/edutec-e/revelec20/anibal20.htm

ULRICH, C, (2008) «Why Web 2.0 is Good for Learning and for Research: Principles and Prototypes", Social Networks \& Web 2.0 - Applications \& Infrastructures for Web 2.0, WWW 2008, ACM 21-25 de Abril, Beiging

URRUTIA, J, (2007) «Las tecnologías de la información y la comunicación en el futuro de la educación, Las TIC en el futuro de la educación», XXII Semana Monográfica Santillana de la Educación, 23 de noviembre de 2007. Disponible en línea en: http://www.oei.es/tic/santillana/urrutia.pdf

WENGER, E, (1999) Communities of Practice. Learning, meaning and identity, Cambridge University Press, Cambridge 